\title{
Slow Relaxations and Bifurcations of the Limit Sets of Dynamical Systems. I. Bifurcations of Limit Sets
}

\author{
A. N. Gorban' ${ }^{1,2^{*}}$ and V. M. Cheresiz ${ }^{3}$ \\ ${ }^{1}$ Department of Mathematics, University of Leicester, University Road, LE1 7RH, United Kingdom \\ ${ }^{2}$ Institute of Computational Modeling, Akademgorodok, Krasnoyarsk, 660036 Russia \\ ${ }^{3}$ Sobolev Institute of Mathematics, pr. Akad. Koptyuga 4, Novosibirsk, 630090 Russia \\ Received August 12, 2008
}

\begin{abstract}
We consider one-parameter semigroups of homeomorphisms depending continuously on the parameters. We study the phenomenon of slow relaxation that consists in anomalously slow motion to the limit sets. We investigate the connection between slow relaxations and bifurcations of limit sets and other singularities of the dynamics. The statements of some of the problems stem from mathematical chemistry.
\end{abstract}

DOI: $10.1134 / \mathrm{S} 1990478910010096$

Key words: one-parameter semigroup of homeomorphisms, slow relaxation, limit set, bifurcation

To the Memory of Sergei L'vovich Sobolev

Consideration of the simplest examples of dynamical systems has already shown that the time for the motion to get into a fixed small neighborhood of the $\omega$-limit set can be arbitrarily large, for example, in view of a delay near an unstable fixed point. Singularities of this kind, which we call slow relaxations, were not studied in topological dynamics separately though the problems related to them arose in various (usually, applied) areas. For instance, there are articles devoted to the removal of the simplest slow relaxations (delays near saddles) under the minimization of functions by relaxation methods (see, for example, [1]).

The impetus to this article was the problem of slow relaxations in chemical kinetics. It was observed in a number of experiments that some chemical (catalytic) systems approach their $\omega$-limit set anomalously slow $[2,3]$. For explaining this phenomenon, some observations about slow "outside" processes (diffusion of reagents into the volume of the catalyst etc.) were involved. In [4,5], it was shown that all these slow relaxations need not be caused by outside processes and can have purely dynamic (or, in the language of the chemists, kinetic) reason. Some mathematical results connected with these problems are announced in our article [6]; their proofs are given in [7]. Another example of slow relaxations in chemistry is given by the induction periods of some chemical reactions (see [8,9]).

In physics, in the study of equilibration processes described by ordinary differential equations, the relaxation time of the system is defined by the quantity $\tau=-(\operatorname{Re} \lambda)^{-1}$, where $\lambda$ is the eigenvalue of the system closest to the imaginary axis. Clearly, this characteristic makes sense only in the description of a small neighborhood of the equilibrium, where the linear approximation holds with adequate accuracy. For nonlinear systems, the choice is nonunique of the time response describing the rate of approximation to the $\omega$-limit set. In this article, we consider the six "relaxation times": the time of the first appearance of the motion in a given neighborhood of its or the full $\omega$-limit set, the time of stay in this neighborhood, and the time of the ultimate entry thereinto.

In topological dynamics, the time responses were usually not considered except for the average time of stay in a domain. More attention is paid to them in the theory of random processes and in the intensively developing theory of small random perturbations of dynamical systems [10]. However, as far as we know, in these areas, the relaxation times were not studied systematically either.

*e-mail: ag153@leicester.ac.uk, gorban@icm.krasn.ru 
This article aims at studying the slow relaxations of dynamical systems. The study is carried out in the spirit of the classical topological dynamics (see [11,12] and also [13]). However, there are some differences: first, we consider not a single dynamical system but a practically more important class of systems depending on the parameters; second, in these systems, the motion is in general defined only for the positive time. This is due to the fact that, in the applications, mainly, in chemical ones, only those notions make sense that are "positively" invariant.

\section{EXTENSION OF SEMIFLOWS TO THE LEFT}

Suppose that we are given a compact metric space $X$ with metric $\rho$, a metric space $K$ with metric $\rho_{K}$, which will play the role of the space of the parameters of the system, and some continuous mapping

$$
f[0, \infty) \times X \times K \rightarrow X .
$$

Moreover, for every $k \in K$ and $t \geq 0$, the mapping $f(t, \cdot, k): X \rightarrow X$ is a homeomorphism of $X$ onto a subset of $X$ and these homeomorphisms form a one-parameter semigroup for each $k \in K$ :

$$
f(0, x, k)=x, \quad f(t+\tau, x, k)=f(t, f(\tau, x, k), k)
$$

for all $t, \tau>0$. In the sequel, we call a semigroup of mappings $f(\cdot, \cdot, k)$ for fixed $k$ a semiflow of homeomorphisms or, for brevity, simply a semiflow; and the mapping (1), a family of semiflows. Obviously, all results valid for the semiflows hold for the dynamical systems; i.e., when each semiflow may be extended "to the left" to the entire axis $(-\infty, \infty)$ to a flow, a one-parameter group of homeomorphisms from $X$ onto $X$.

It is clear that, in general, for fixed $x$ and $k$, the mapping $f(\cdot, x, k): t \rightarrow f(t, x, k)$ may be extended to some negative $t$ with preservation of the semigroup property (2). Indeed, for fixed $x$ and $k$, consider the set of all negative $t$ for each of which there is $q_{t} \in X$ such that $f\left(t, q_{t}, k\right)=x$. Let $T(x, k)$ be the supremum of this set:

$$
T(x, k)=\sup \left\{t \geq 0 \mid \exists q_{t} \in X: f\left(t, q_{t}, k\right)=x\right\} .
$$

For given $t, x$, and $k$, the point $q_{t}$, if it exists, is defined uniquely since the mapping

$$
f(t, \cdot, k): X \rightarrow X
$$

is a homeomorphism. Put $f(-t, x, k)=q_{t}$. If $f(-t, x, k)$ is defined then

$$
f(-\tau, x, k)=f(t-\tau, f(-t, x, k), k)
$$

is also defined for all $\tau$ with $0 \leq \tau \leq t$. Suppose that $T(x, k)<\infty$ and numbers $t_{n}$ are such that $t_{n}<T(x, k)$ for all $n$ and $t_{n} \rightarrow T(x, k)$ as $n \rightarrow \infty$. From the sequence $\left\{f\left(-t_{n}, x, k\right)\right\}$, choose a subsequence converging to some $q^{*} \in X$ and denote it by $\left\{q_{j}\right\}$, and the corresponding times, by $-t_{j}$; so that $q_{j}=f\left(-t_{j}, x, k\right)$. Since $f$ is continuous, we infer $f\left(t_{j}, q_{j}, k\right) \rightarrow f\left(T(x, k), q^{*}, k\right)$; therefore, $f\left(T(x, k), q^{*}, k\right)=x$. Hence, $f(-T(x, k), x, k)=q^{*}$.

Thus, for fixed $x$ and $k$, we have the mapping $f$ on $[-T(x, k), \infty)$ if $T(x, k)$ is finite, and on the entire axis $(-\infty, \infty)$ otherwise. Let $S$ be the set of all triples $(t, x, k)$ on which $f$ is now defined. This extended mapping possesses the subgroup property as follows:

Proposition 1. If $(\tau, x, k)$ and $(t, f(\tau, x, k), k) \in S$ then

$$
f(t, f(\tau, x, k), k)=f(t+\tau, x, k) .
$$

Thus, if the left-hand side of (3) makes sense then so does the right-hand side and the equality holds.

Conversely, if $(t+\tau, x, k)$ and $(\tau, x, k) \in S$ then $(t, f(\tau, x, k), k) \in S$ and (3) holds. In other words, if in (3) the right-hand side and $f(\tau, x, k)$ in the left-hand side are defined then the entire left-hand side makes sense and, hence, (3) holds again. 
Proof. Since $k$ is assumed fixed; therefore, for brevity, we do not mention it in the formulas below. Note that if at least one of the numbers $t$ and $\tau$ is zero then we have nothing to prove. Therefore, assume them nonzero and consider all the four logically possible variants of their sign combinations. If they are both positive then all our assertions are valid by definition. However, in the three remaining variants, it will be convenient to assume that $t>0$ and $\tau>0$ and replace in (3) one of these numbers or both of them with the opposite numbers.

Variant I. $f(t, f(-\tau, x))=f(t-\tau, x)$.

We first stress that if $t=\tau$ then all assertions related to Variant I are obvious. Thus, we are left with only the two possibilities that we will now discuss:

(a) Suppose first that $t>\tau$. In this case, the hypotheses of both our assertions, direct and converse, in view of the inequalities $t>0$ and $t-\tau>0$, are reduced to the condition that $f(-\tau, x)$ must be defined. If it is so then

$$
f(t, f(-\tau, x))=f((t-\tau)+\tau, f(-\tau, x))=f(t-\tau, f(\tau, f(-\tau, x)))=f(t-\tau, x),
$$

and, hence, the first case is exhausted.

(b) Suppose now that $\tau>t$. Suppose that $f(-\tau, x)$ is defined. Then both the left-hand side (since $t>0$ ) and the right-hand side of the inequality ( since $t-\tau>-\tau$ ) are defined. Here

$$
f(\tau-t, f(t, f(-\tau, x)))=f(\tau-t+t, f(-\tau, x))=x,
$$

whence, by definition, $f(-(\tau-t), x)=f(t, f(-\tau, x))$. Thus, the second case is studied:

Variant II. $f(-t, f(\tau, x))=f(-t+\tau, x)$.

As above, the inequality needs no proof for $t=\tau$.

(a) Suppose now that $\tau>t$. By definition, $f(-\tau, f(\tau, x))$ makes sense; and, since $-t>-\tau$, so does $f(-t, f(\tau, x))$ all the more. Moreover, $f(-t+\tau, x)$ is defined since $-t+\tau>0$. In other words, both sides of the equality in Variant II are defined. As for the validity of equality, it suffices to observe that $f(t, f(-t+\tau, x))=f(\tau, x)$; and, hence, $f(-t+\tau, x)=f(-t, f(\tau, x))$ by the definition of $f$ for negative times.

(b) Suppose to the contrary that $t>\tau$. Assume that the left-hand side is defined in the quality of Variant II. Noticing that $t-\tau>0$, we make use of the equality of Variant I, by which

$$
f(t-\tau, f(-t, f(\tau, x)))=f(-\tau, f(\tau, x))=x .
$$

Therefore, $f(-(t-\tau), x)=f(-t+\tau)$ makes sense and is equal to $f(-t, f(\tau, x))$. Conversely, suppose that $f(-t+\tau, x)$ is defined. Since, for $\tau>0, f(\tau, x)$ is defined a priori, we may once again apply the equality of Variant I and infer $f(t, f(-t+\tau, x))=f(\tau, x)$. This means that $f(-t, f(\tau, x))$ is defined and equal to $f(-t+\tau, x)$.

Variant III. $f(-t, f(-\tau, x))=f(-t-\tau, x)$.

Suppose that the left-hand side of this equality is defined. From Variant I it follows that

$$
f(t+\tau, f(-t, f(-\tau, x)))=f(\tau, f(-\tau, x))=x .
$$

Consequently, $f(-t-\tau, x)$ is defined and equal to $f(-t, f(-\tau, x))$. Conversely, suppose that $f(-\tau, x)$ and $f(-t-\tau, x)$ are defined. By Variant I, we then have $f(t, f(-t-\tau, x))=f(-\tau, x)$ and, hence, $f(-t, f(-\tau, x))$ is defined and equal to $f(-t-\tau, x)$. Proposition 1 is proved.

Proposition 2. $S$ is closed in the product $(-\infty, \infty) \times X \times K$, and $f: S \rightarrow X$ is continuous.

Proof. Let $\langle a, \infty)$ denote the interval $[a, \infty)$ if $a$ is finite and the entire real axis $(-\infty, \infty)$ when $a=-\infty$. Suppose that $t_{n} \rightarrow t^{*}, x_{n} \rightarrow x^{*}, k_{n} \rightarrow k^{*}$, and $t_{n}$ lies in $\left\langle-T\left(x_{n}, k_{n}\right), \infty\right)$. We aim at verifying that $t^{*}$ is in $\left\langle-T\left(x^{*}, k^{*}\right), \infty\right)$ and also $f\left(t_{n}, x_{n}, k_{n}\right) \rightarrow f\left(t^{*}, x^{*}, k^{*}\right)$. If all $t_{n} \geq 0$ then this follows from continuity of $f$ on $[0, \infty) \times X \times K$. Consider now the case when all $t_{n} \leq 0$. A sequence in a compact metric space always has at least one partial limit; it converges if and only if such a limit is unique. Let $q^{*}$ be one of the partial limits of the sequence $q_{n}=f\left(t_{n}, x_{n}, k_{n}\right)$; i.e., there are numbers $n_{i}$ such that $n_{i} \rightarrow \infty$ and $q_{n_{i}} \rightarrow q^{*}$ as $i \rightarrow \infty$. Then the sequence $x_{n_{i}}=f\left(-t_{n_{i}}, q_{n_{i}}, k_{n_{i}}\right)$, on the one hand, converges to $f\left(-t^{*}, q^{*}, k^{*}\right)$ and, on the other hand, to $x^{*}$. Thus, $f\left(-t^{*}, q^{*}, k^{*}\right)=x^{*}$. This means that, first, $t^{*}$ is contained in $\left\langle-T\left(x_{n}, k_{n}\right), \infty\right)$ and $f\left(t^{*}, x^{*}, k^{*}\right)=q^{*}$; and, second, $f\left(t^{*}, x^{*}, k^{*}\right)$ is the only partial limit of the sequence $q_{n}$, which thus converges thereto. The proposition is proved. 
Throughout the sequel, we call the mapping $f(\cdot, x, k):\langle-T(x, k), \infty) \rightarrow X$ the $(x, k)$-motion; it is natural to call its image the $(x, k)$-trajectory. Sometimes we will speak of the images of the intervals $\langle-T(x, k), 0]$ and $[0, \infty)$ calling them the negative and positive $(x, k)$-semitrajectories. We call the $(x, k)$-motion and its trajectory entire if $T(x, k)=\infty$.

Suppose that the $\left(x_{n}, k_{n}\right)$-motion is defined at least on $\left[-t_{n}, \infty\right)$, where $t_{n}>0$. Assume that

$$
\left(x_{n}, k_{n}\right) \rightarrow\left(x^{*}, k^{*}\right), \quad t_{n} \rightarrow t^{*}, \quad 0<t^{*}<\infty .
$$

Then the $\left(x^{*}, k^{*}\right)$-motion is defined on $\left[-t^{*}, \infty\right)$. In particular, if all $\left(x_{n}, k_{n}\right)$-motions are defined on some common interval of the type under consideration, the $\left(x^{*}, k^{*}\right)$-motion is defined on the same interval. If $t_{n} \rightarrow \infty$ then the $\left(x^{*}, k^{*}\right)$-motion is defined on the entire real axis; i.e., it is an entire motion. In particular, if all $\left(x_{n}, k_{n}\right)$-motions are integer then so is the $\left(x^{*}, k^{*}\right)$-motion. All that follows immediately from the closedness of the set $S$ on which the extended mapping $f$ is defined.

In the conclusion of the section, we focus the reader's attention on the two circumstances closely connected with the problems under consideration. Our first remark fits well under the heading "Do Not Think." Suppose as above that $\left(x_{n}, k_{n}\right) \rightarrow\left(x^{*}, k^{*}\right)$. The duration of the "prehistories" of the corresponding motions are defined by the quantities $T\left(x_{n}, k_{n}\right)$ and $T\left(x^{*}, k^{*}\right)$. Our main conclusion about the connection between these times may be expressed in one simple sentence: If a number $\tau>0$ is such that $T\left(x_{n}, k_{n}\right) \geq \tau$ for all $n$ then $T\left(x^{*}, k^{*}\right) \geq \tau$. There appears the question of whether the converse holds, of course, in the sense natural for this situation, namely: if it s known that $T\left(x^{*}, k^{*}\right)>\tau$ then can we assume that $T\left(x_{n}, k_{n}\right)>\tau$ for all sufficiently large $n$ ? To debunk any illusion about that, represent the answer in its extremely negative form: It can happen that $T\left(x_{n}, k_{n}\right) \equiv 0$ but, at the same time, $T\left(x^{*}, k^{*}\right)=\infty$.

The second simple remark already has a "positive" nature. Suppose once again that $\left(x_{n}, k_{n}\right) \rightarrow$ $\left(x^{*}, k^{*}\right)$ and all $\left(x_{n}, k_{n}\right)$-motions and, hence, the $\left(x^{*}, k^{*}\right)$-motion are defined on $[a, b]$. Then, as is easy to understand, the $\left(x_{n}, k_{n}\right)$-motions converge to the $\left(x^{*}, k^{*}\right)$-motion uniformly on $[a, b]$ in the sense that $f\left(t, x_{n}, k_{n}\right) \rightarrow f\left(t, x^{*}, k^{*}\right)$ uniformly over all $t$ in $a \leq t \leq b$. This is immediate from the continuity of $f$ and the compactness of the interval $[a, b]$.

\section{LIMIT SETS}

Definition 1. A point $p$ is called an $\omega$-limit point of the $(x, k)$-motion if there exists a sequence $t_{n} \rightarrow \infty$ for which $f\left(t_{n}, x, k\right) \rightarrow p$. All these points constitute the $\omega$-limit set of the $(x, k)$-motion, which we will denote by $\omega(x, k)$. If the $(x, k)$-motion is entire then we may speak of the partial limits of the mapping $f(t, x, k)$ as $t \rightarrow-\infty$ and, by analogy to the above, define $\alpha$-limit points of such a motion and its $\alpha$-limit set $\alpha(x, k)$.

Note that, by the compactness of the space $X$, each motion has at least one $\omega$-limit point, and so its $\omega$-limit set is always nonempty. Of course, the same holds for the $\alpha$-limit sets of entire motions.

Definition 2. We call a nonempty set $W \subset X k$-invariant if, for each $x \in W$, the corresponding $(x, k)$-motion is entire and the whole of its trajectory lies in $W$. Analogously, a set $V \subset X$ is $(k, \omega)$ invariant or positively $k$-invariant if it is nonempty and $f(t, x, k) \in V$ for any $t>0$ and $x \in V$.

Proposition 3. The limit set $\omega(x, k)$ of every $(x, k)$-motion is $k$-invariant. The same holds for the limit set $\alpha(x, k)$ of an entire $(x, k)$-motion.

Proof. Suppose that $p \in \omega(x, k)$. Find a sequence $t_{n} \rightarrow \infty$ for which $x_{n}=f\left(t_{n}, x, k\right) \rightarrow p$. Note that the $\left(x_{n}, k\right)$-motion is defined at least on $\left[-t_{n}, \infty\right)$. Therefore, as was observed above, the $(p, k)$-motion is defined everywhere on the real axis; i.e., it is entire. Show that the entire $(p, k)$-trajectory consists of $\omega$-limit points of the $(x, k)$-motion. Choose a point $f(\tau, x, k)$ on this trajectory. Since $t_{n} \rightarrow \infty$, the values $f\left(\tau+t_{n}, x, k\right)$ are defined for all sufficiently large $n$. They are equal to $f\left(\tau, x_{n}, k\right)$ by Proposition 1 , and, hence, by the condition $x_{n} \rightarrow p$ and the continuity of the extended mapping $f$ established in Proposition 2, tend to $f(\tau, p, k)$. Thus, as was asserted, $f(\tau, p, k) \in \omega(p, k)$.

Suppose now that the $(x, k)$-motion is entire and $q \in \alpha(x, k)$. Choose a sequence $t_{n} \rightarrow-\infty$ for which $x_{n}=f\left(t_{n}, x, k\right) \rightarrow q$. Clearly, all $\left(x_{n}, k\right)$-motions are entire too. Fix an arbitrary moment $\tau$ and note that $f\left(\tau+t_{n}, x, k\right)=f\left(\tau, x_{n}, k\right) \rightarrow f(\tau, q, k)$ thanks to the semigroup property and the continuity of $f$. Since $\tau+t_{n} \rightarrow-\infty$, the point $f(\tau, q, k)$ is $\alpha$-limit for the $(x, k)$-motion. The proposition is proved. 


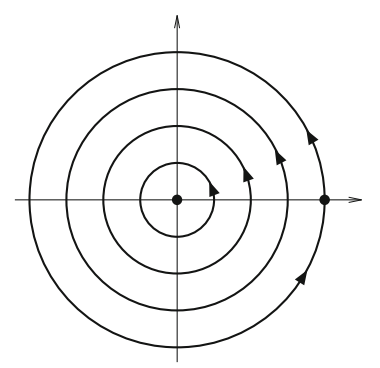

Fig. 1. The phase portrait of the system of Example 1

Proposition 4. The set $\omega(x, k)$ is closed and connected for every $(x, k)$-motion. The limit set $\alpha(x, k)$ of an entire $(x, k)$-motion possesses the same properties.

We omit the proof because it coincides almost verbatim with the proof of the analogous assertions in $[12$, p. 358-362].

In the sequel, apart from the $\omega$-limit sets of individual motions, we consider the full $\omega$-limit set

$$
\omega(k)=\bigcup_{x \in X} \omega(x, k) .
$$

This set is of course $k$-invariant, since is a union of $k$-invariant sets, but need not be closed.

Example 1 (the nonclosedness of a complete $\omega$-limit set). Consider the planar system in the disk $x^{2}+y^{2} \leq 1$ defined by the equation

$$
\dot{x}=y(x-1), \quad \dot{y}=-x(x-1) .
$$

The complete $\omega$-limit set consists of the open disk $\left\{x^{2}+y^{2}<1\right\}$ and the point $(0,1)$. It is not closed. Its closure coincides with the circle, the boundary consists of two trajectories: the fixed point $(1,0) \in \omega$ and the loop $\left\{x^{2}+y^{2}=1, x \neq 1\right\}$ disjoint with $\omega$. The phase trajectories of this system are shown in Fig. 1. In the domain $\left\{0<x^{2}+y^{2}<1\right\}$, they are all concentric circles with center the fixed origin.

For studying the slow relaxations of dynamical systems, we need the two classes of sets composed of the $\omega$-limit sets of motions, namely:

$$
\Omega(x, k)=\left\{\omega\left(x^{\prime}, k\right) \mid \omega\left(x^{\prime}, k\right) \subset \omega(x, k)\right\}, \quad \Omega(k)=\{\omega(x, k) \mid x \in X\} .
$$

Thus, $\Omega(x, k)$ consists of all subsets $\omega(x, k)$ that serve as the $\omega$-limit sets of some motions. The class $\Omega(k)$ consists of the $\omega$-limit sets of all kinds of motions of the system.

\section{CONVERGENCES IN HYPERSPACES}

In the second part of our article, we study the connection between the slow relaxations of dynamical systems and bifurcations; i.e., the violations of the continuous dependence of the sets $\omega(x, k)$ and $\omega(k)$ and the classes $\Omega(x, k)$ and $\Omega(k)$ on $x$ and $k$. Here we only define some kinds of convergences in the spaces of sets and the classes of sets necessary for this work and consider the mappings continuous with respect to these convergences. One of the notions of continuity that we use is well known. This is lower semicontinuity (see $[14, \S 18]$ and $[15, \S 43]$ ). The other two are somewhat more "exotic."

Denote the class of all nonempty subsets of $X$ by $B(X)$, which is the hyperspace of $X$. By the distance form a point $x \in X$ to a set $q \in B(X)$ we mean the quantity

$$
\rho^{*}(x, q)=\inf _{y \in q} \rho(x, y) .
$$

We first introduce the following measure of proximity in $B(X)$ : Given some sets $p$ and $q$ in $B(X)$, we put

$$
d(p, q)=\sup _{x \in p} \rho^{*}(x, q) .
$$

The function $d(p, q)$ is in general nonsymmetric and is a "half" of the well-known Hausdorff metric $[14$, p. 223] in which the distance between two sets $p$ and $q$ is the greatest of the quantities $d(p, q)$ 
and $d(q, p)$. Define convergence in $B(X)$ with the help of the above proximity measure: Let $q_{n}$ be a sequence of points in $B(X)$. We say that it $d$-converges to $p \in B(X)$ if $d\left(p, q_{n}\right) \rightarrow 0$. As is easy to understand, $d$-convergence defines a topology in $B(X)$ with countable base at each point, and continuity in this topology coincides with $d$-continuity (the $\lambda$-topology [14, p. 183]). In this topology, a basis of neighborhoods of a point $p \in B(X)$ is given, for example, by the family of sets

$$
\{q \in B(X) \mid d(p, q)<1 / n\}, \quad n \geq 1 .
$$

The conditions that guarantee that this is indeed a topology are easily verifiable, since, in spite if its asymmetry, the function $d$ satisfies the triangle inequality $d(p, s) \leq d(p, q)+d(q, s)$. For this conditions, see [16, p. 19-20].

Another measure of proximity between sets $p$ and $q$ in $B(X)$ that we need is defined as

$$
r(p, q)=\inf _{x \in p} \rho^{*}(x, q) .
$$

By analogy to the above, the $r$-convergence of $q_{n}$ to $p$ means that $r\left(p, q_{n}\right) \rightarrow 0$. Note that $r$-convergence does not define a topology in $B(X)$. To prove that, make use of the following obvious property of convergence in topological spaces: if $p_{i}=p, q_{i}=q, s_{i}=s$ are constant sequences of points, and $p_{i} \rightarrow q$, $q_{i} \rightarrow s$ then $p_{i} \rightarrow s$. This property does not hold for $r$-convergence. To construct an example, it suffices to take two points $x, y \in X, x \neq y$, and put $p=\{x\}, q=\{x, y\}, s=\{y\}$. Then $r(p, q)=r(q, p)=0$, and $r(p, s) \neq 0$. Therefore, $p_{i} \rightarrow q, q_{i} \rightarrow s$, but $p_{i} \not \rightarrow s$; hence, that the $r$-convergence does not define a topology for any metric space $X$ that is not a point.

In the sequel, we need the following two criteria for the convergence of sequences in $B(X)$. In connection of the first see, for example, [14, p. 184, the generalized Heine condition]; the second follows directly from the definition of $r$-proximity.

Proposition 5. A sequence $q_{n} \in B(X)$ tends to $p \in B(X)$ in the sense of d-convergence if and only if $\rho^{*}\left(x, q_{n}\right) \rightarrow 0$ for all $x \in p$.

Proof. Let $q_{n} \rightarrow p$. Then $d\left(p, q_{n}\right) \rightarrow 0$; and, hence, $\rho^{*}\left(x, q_{n}\right) \rightarrow 0$ for every $x \in p$ since the supremum of these numbers over $x$ vanishes. Suppose to the contrary that $\rho^{*}\left(x, q_{n}\right) \rightarrow 0$ for every $x \in p$. We must prove that $d\left(p, q_{n}\right) \rightarrow 0$. This follows from the compactness of $X$. Indeed, suppose to the contrary that $d\left(p, q_{n}\right) \nrightarrow 0$. Then, passing if necessary to a subsequence, we may assume that, for some $\varepsilon>0$, the inequality $d\left(p, q_{n}\right)>\varepsilon$ holds for all $n$. In this case, there is a sequence $x_{n} \in p$ such that $\rho^{*}\left(x_{n}, q_{n}\right)>\varepsilon$. Since $X$ is compact, $x_{n}$ may be assumed convergent, and then there exists a number $N$ such that $\rho\left(x_{n}, x_{N}\right)<\varepsilon / 2$ for $n>N$. Consequently, if $y \in q_{n}$ and $n>N$ then

$$
\rho\left(x_{N}, y\right)>\rho\left(x_{n}, y\right)-\varepsilon / 2>\varepsilon-\varepsilon / 2=\varepsilon / 2 .
$$

Therefore, $\rho^{*}\left(x_{N}, q_{n}\right)>\varepsilon / 2$. We arrived at a contradiction because $x_{N} \in p$ and, hence, $\rho^{*}\left(x_{N}, q_{n}\right) \rightarrow 0$ as $n \rightarrow \infty$. The proof is complete.

Proposition 6. A sequence $q_{n} \in B(X)$ tends to $p \in B(X)$ in the sense of $r$-convergence if and only if there exist such $y_{n} \in q_{n}$ and $x_{n} \in p$ that $\rho\left(x_{n}, y_{n}\right) \rightarrow 0$.

Let $B(B(X))$ denote the class of all nonempty subclasses in $B(X)$. Our nearest aim is to introduce a proximity measure in this class. To simplify exposition, given $p \in B(X)$ and $Q \in B(B(X))$ put

$$
r^{*}(p, Q)=\inf _{q \in Q} r(p, q) .
$$

As a measure of proximity between the classes $P$ and $Q$ in $B(B(X))$, take the quantity

$$
D(P, Q)=\sup _{p \in P} r^{*}(p, Q) .
$$

Note that (5) repeats (4) with the only difference that it involves $r$ instead of $\rho$. We may slightly simplify (5) by introducing the following notations: Suppose that $Q \in B(B(X))$. Put $S Q=\bigcup_{q \in Q} q$. Then $S Q \in B(X)$ and

$$
D(P, Q)=\sup _{p \in P} r(p, S Q)
$$


We say that a sequence $Q_{n}$ in $B(B(X)) \quad D$-converges to $P \in B(B(X))$ if $D\left(P, Q_{n}\right) \rightarrow 0$. Like the $r$-convergence, $D$-convergence does not define a topology. This may be proved similarly to how it was done for $r$-convergence. Namely, suppose that $x, y \in X x \neq y$. Put $P=\{\{x\}\}, Q=\{\{x, y\}\}$, and $R=\{\{y\}\}$. Then $D(Q, P)=D(R, Q)=0, P_{i} \rightarrow Q, Q_{i} \rightarrow R$. Moreover, $D(R, P)=\rho(x, y) \neq 0$, and so $P_{i} \nrightarrow R$.

Before passing to a criterion of $D$-convergence, prove the following topological lemma:

Lemma 1. Consider two sequences $p_{n}$ and $q_{n}$ of subsets of $X$. Suppose that, for some $\varepsilon>0$, the inequality $r\left(p_{n}, q_{n}\right)>\varepsilon$ holds for every $n$. Then, for every $\gamma<\varepsilon$, there exists an infinite set of indices $J$ such that $r\left(p_{k}, q_{l}\right)>\gamma$ for all $k, l \in J$.

Proof. Putting $\delta=(\varepsilon-\gamma) / 4$, choose a finite $\delta$-net $M$ of $X$; and, to each $p \subset X$, assign the set

$$
p^{*}=\left\{m \in M \mid \rho^{*}(m, p) \leq \delta\right\} .
$$

As is easy to understand, every two subsets $p$ and $q$ in $X$ satisfy the inequality $\left|r(p, q)-r\left(p^{*}, q^{*}\right)\right| \leq 2 \delta$. Therefore, $r\left(p_{n}^{*}, q_{n}^{*}\right)>\varepsilon-2 \delta$ for all $n$. Since $M$ is finite, the number of distinct pairs in the infinite sequence $p_{n}^{*}, q_{n}^{*}$ is in fact finite. Consequently, there exist an infinite set of indices $J$ and a number $N$ such that $p_{n}^{*}=p_{N}^{*}$ and $q_{n}^{*}=q_{N}^{*}$ for all $n \in J$. Thus, if $k, l \in J$ then $r\left(p_{k}^{*}, q_{l}^{*}\right)=r\left(p_{N}^{*}, q_{N}^{*}\right)>\varepsilon-2 \delta$. This obviously implies that $r\left(p_{k}, q_{l}\right)>\varepsilon-4 \delta=\gamma$. The lemma is proved.

Proposition 7. A sequence $Q_{n} \in B(B(X))$ converges to $P \in B(B(X))$ in the sense of $D$ convergence if and only if $r^{*}\left(p, Q_{n}\right) \rightarrow 0$ for every $p \in P$.

Proof. This is obvious in one direction: if $Q_{n} \rightarrow P$ then, by definition, $D\left(P, Q_{n}\right) \rightarrow 0$ and, all the more, $r^{*}\left(p, Q_{n}\right) \rightarrow 0$ for every $p \in P$. Assume now that, on the contrary, $r^{*}\left(p, Q_{n}\right) \rightarrow 0$ for every $p \in P$. If, moreover, $D\left(P, Q_{n}\right) \nrightarrow 0$ then we may assume that $D\left(P, Q_{n}\right)>\varepsilon$ for some $\varepsilon>0$ and all $n$. In this case, by (6), there exist points $p_{n} \in P$ for which $r\left(p_{n}, S Q_{n}\right)>\varepsilon$. By Lemma 1, there exist $\gamma>0$ and a number $N$ such that $r\left(p_{N}, S Q_{n}\right)>\gamma$ for arbitrarily large $n$. Thus, the sequence $r^{*}\left(p_{N}, Q_{n}\right)=$ $r\left(p_{N}, S Q_{n}\right)$ does not tend to zero. With this contradiction, we finished the proof of the assumption.

Throughout the sequel, unless otherwise specified, by convergence in $B(X)$ we mean $d$-convergence; and convergence in $B(B(X)), D$-convergence. Of course, the semicontinuity of functions is also considered with respect to these convergences.

\section{BIFURCATIONS OF LIMIT SETS}

Definition 3. We say that the family of semiflows (1) admits:

(a) $\omega(x, k)$-bifurcations if $\omega(x, k)$ is not a $d$-continuous function in $X \times K$;

(b) $\omega(k)$-bifurcations if $\omega(k)$ is not a $d$-continuous function in $K$;

(c) $\Omega(x, k)$-bifurcations if $\Omega(x, k)$ is not a $D$-continuous function in $X \times K$;

(d) $\Omega(k)$-bifurcations if $\Omega(k)$ is not a $D$-continuous function in $K$.

By the bifurcation points we mean the points in $X \times K$ or $K$ at which the $d$-continuity fails of $\omega(x, k)$ and $\omega(k)$ or the $D$-continuity fails of $\Omega(x, k)$ and $\Omega(k)$. The discontinuities of these functions that we consider could be called blow-ups of limit sets (cf. a blow-up of the set of nonwandering points in differential dynamics [17, 6.3 , p. 185-192], which is, however, a violation of upper semicontinuity).

Proposition 8. (a) If (1) admits $\Omega(k)$-bifurcations then it admits $\Omega(x, k)-, \omega(k)$-, and $\omega(x, k)$ bifurcations. (b) If the system admits $\Omega(x, k)$-bifurcations then it admits $\omega(x, k)$-bifurcations. (c) If there exist $\omega(k)$-bifurcations then there are also $\omega(x, k)$-bifurcations.

It is convenient to illustrate this proposition by the scheme in Fig. 2 in which the word "bifurcation" is omitted. 


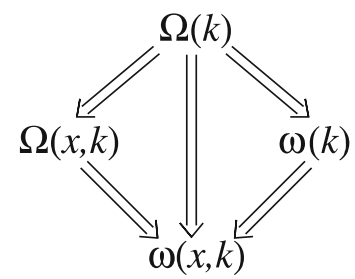

Fig. 2. A scheme of bifurcations (see Proposition 8)

Proof. We begin with (c). Suppose that, at a point $k^{*} \in K$, system (1) has an $\omega(k)$-bifurcation. By Proposition 5, this means that there exist such $\varepsilon>0, x^{*} \in \omega\left(k^{*}\right)$, and a sequence $k_{n} \in K$ converging to $k^{*}$ for which $\rho^{*}\left(x^{*}, \omega\left(k_{n}\right)\right)>\varepsilon$ for all $n$. The point $x^{*}$ belongs to the limit set $\omega\left(x_{0}, k^{*}\right)$ of some point $x_{0} \in X$. Note that $\omega\left(x_{0}, k_{n}\right) \subset \omega\left(k_{n}\right)$. Consequently, $\rho^{*}\left(x^{*}, \omega\left(x_{0}, k_{n}\right)\right)>\varepsilon$. Therefore, $\omega\left(x_{0}, k_{n}\right)$ does not converge to $\omega\left(x_{0}, k^{*}\right)$. Thus, $\left(x_{0}, k^{*}\right)$ is an $\omega(x, k)$-bifurcation point.

Prove (b). Suppose that, at a point $\left(x^{*}, k^{*}\right) \in X \times K$, our system has an $\Omega(x, k)$-bifurcation. Then, by Proposition 8 , there are $\varepsilon>0, \omega\left(x_{0}, k^{*}\right) \subset \omega\left(x^{*}, k^{*}\right)$, and a sequence $\left(x_{n}, k_{n}\right)$ converging to $\left(x^{*}, k^{*}\right)$ such that $r\left(\omega\left(x_{0}, k^{*}\right), S \Omega\left(x_{n}, k_{n}\right)\right)>\varepsilon$ for each $n$. This means that $r\left(\omega\left(x_{0}, k^{*}\right), \omega\left(x_{n}, k_{n}\right)\right)>\varepsilon$ and, hence, $\rho^{*}\left(\xi, \omega\left(x_{n}, k_{n}\right)\right)>\varepsilon$ for every $\xi \in \omega\left(x_{0}, k^{*}\right)$. Since $\xi \in \omega\left(x^{*}, k^{*}\right)$, this implies that $\left(x^{*}, k^{*}\right)$ is an $\omega(x, k)$-bifurcation point for $(1)$.

Prove the assertions of (a). Let $k^{*} \in K$ be an $\Omega(k)$-bifurcation point for (1). Then there exist $\varepsilon>0$ and a sequence of points $k_{n} \in K$ converging to $k_{n} \rightarrow k^{*}$ for which $D\left(\Omega\left(k^{*}\right), \Omega\left(k_{n}\right)\right)>\varepsilon$ for each $n$; i.e., by (6), for each $n$, there exists $x_{n} \in X$ such that $r\left(\omega\left(x_{n}, k^{*}\right), \omega\left(k_{n}\right)\right)>\varepsilon$. By Lemma 1 , there are $\gamma>0$ and a natural number $N$ such that the inequality $r\left(\omega\left(x_{N}, k^{*}\right), \omega\left(k_{n}\right)\right)>\gamma$ holds for an infinite set of indices $J$ for all $n \in J$. All the more, $r\left(\omega\left(x_{N}, k^{*}\right), \omega\left(x_{N}, k_{n}\right)\right)>\gamma$. Consequently, there are $\Omega(x, k)$ bifurcations; namely: on the one hand, $\left(x_{N}, k_{n}\right) \rightarrow\left(x_{N}, k^{*}\right)$ as $n$ tends to $\infty$ remaining within $J$; and on the other hand, the quantity

$$
D\left(\Omega\left(x_{N}, k^{*}\right), \Omega\left(x_{N}, k_{n}\right)\right)=\sup \left\{r\left(\omega\left(x, k^{*}\right), \omega\left(x_{N}, k_{n}\right)\right) \mid \omega\left(x, k^{*}\right) \in \Omega\left(x_{N}, k^{*}\right)\right\}
$$

is greater than $r\left(\omega\left(x_{N}, k^{*}\right), \omega\left(x_{N}, k_{n}\right)\right)>\gamma$, so that $\left(x_{N}, k^{*}\right)$ is an $\Omega(x, k)$-bifurcation point.

Taking the already-established validity of the implications (b) and (c) into account, we are only left with proving that if there are $\Omega(k)$-bifurcations then there are $\omega(k)$-bifurcations. Prove this. Let $k^{*} \in K$ be an $\Omega(k)$-bifurcation point for (1). Then, as we have just proved, there exist a point $x^{*} \in X$ $\left(x_{N}\right)$ and a sequence of points $k_{n} \in K$ tending to $k^{*}$ for which $r\left(\omega\left(x^{*}, k^{*}\right), \omega\left(k_{n}\right)\right)>\gamma$. All the more, $\rho^{*}\left(\xi, \omega\left(k_{n}\right)\right)>\gamma$ for every $\xi \in \omega\left(x^{*}, k^{*}\right)$. This implies that $d\left(\omega\left(k^{*}\right), \omega\left(k_{n}\right)\right)>\gamma$ and, hence, an $\Omega(k)$ bifurcation of the system occurs at $k^{*}$. The proposition is proved.

Proposition 9. A system has $\Omega(x, k)$-bifurcations if and only if $\omega(x, k)$ is not $r$-continuous on $X \times K$.

Proof. Suppose that the system has $\Omega(x, k)$-bifurcations. Then there exist a point $\left(x^{*}, k^{*}\right) \in X \times K$, a sequence $\left(x_{n}, k_{n}\right) \in X \times K$ such that $\left(x_{n}, k_{n}\right) \rightarrow\left(x^{*}, k^{*}\right)$, and a number $\varepsilon>0$, for which the inequality $D\left(\Omega\left(x^{*}, k^{*}\right), \Omega\left(x_{n}, k_{n}\right)\right)>\varepsilon$ holds for each $n$. This means that, for each $n$, there exists $x_{n}^{*} \in X$ for which $\omega\left(x_{n}^{*}, k^{*}\right) \subset \omega\left(x^{*}, k^{*}\right), r\left(\omega\left(x_{n}^{*}, k^{*}\right), \omega\left(x_{n}, k_{n}\right)\right)>\varepsilon$. Lemma 1 implies the existence of $\gamma>0$, some natural $N$, and an infinite set of indices $J$ such that $r\left(\omega\left(x_{N}, k^{*}\right), \omega\left(x_{n}, k_{n}\right)\right)>\gamma$ for $n \in J$. Let $x_{0}^{*}$ be an arbitrary point in $\omega\left(x_{N}^{*}, k^{*}\right)$. As we have already observed, the $\left(x_{0}^{*}, k^{*}\right)$-trajectory lies in $\omega\left(x_{N}^{*}, k^{*}\right)$, and, by the closedness of this set, $\omega\left(x_{0}^{*}, k^{*}\right) \subset \omega\left(x_{N}^{*}, k^{*}\right)$. Therefore,

$$
r\left(\omega\left(x_{0}^{*}, k^{*}\right), \omega\left(x_{n}, k_{n}\right)\right)>\gamma, \quad n \in J .
$$

Since $x_{0}^{*} \in \omega\left(x^{*}, k^{*}\right)$, there exists a sequence $t_{i}>0$ such that

$$
t_{i} \rightarrow \infty, \quad f\left(t_{i}, x^{*}, k^{*}\right) \rightarrow x_{0}^{*} \quad \text { as } \quad i \rightarrow \infty .
$$

Using the continuity of $f$, for each $i$, choose a number $n(i) \in J$ such that

$$
\rho\left(f\left(t_{i}, x^{*}, k^{*}\right), f\left(t_{i}, x_{n(i)}, k_{n(i)}\right)\right)<1 / i .
$$


Let $\left.f\left(t_{i}, x_{n(i)}, k_{n(i)}\right)=x_{i}^{\prime}, k_{n(i)}\right)=k_{i}^{\prime}$. Note that $\omega\left(x_{i}^{\prime}, k_{i}^{\prime}\right)=\omega\left(x_{n(i)}, k_{n(i)}\right)$. Therefore,

$$
r\left(\omega\left(x_{0}^{*}, k^{*}\right), \omega\left(x_{i}^{\prime}, k_{i}^{\prime}\right)\right)>\gamma \text { for each } i .
$$

Since $\left(x_{i}^{\prime}, k_{i}^{\prime}\right) \rightarrow\left(x_{0}^{*}, k^{*}\right)$, the function $\omega(x, k)$ does not satisfy the condition of $r$-continuity at the point $\left(x_{0}^{*}, k^{*}\right)$.

We stress that an $\Omega(x, k)$-bifurcation may fail to be an $r$-discontinuity point.

Suppose now that $\omega(x, k)$ is not $r$-continuous in $X \times K$. Then there exist $\left(x^{*}, k^{*}\right) \in X \times K$, a sequence of points $\left(x_{n}, k_{n}\right) \in X \times K,\left(x_{n}, k_{n}\right) \rightarrow\left(x^{*}, k^{*}\right)$, and $\varepsilon>0$ for which $r\left(\omega\left(x^{*}, k^{*}\right), \omega\left(x_{n}, k_{n}\right)\right)>$ $\varepsilon$ for each $n$. By (6), this implies $D\left(\Omega\left(x^{*}, k^{*}\right), \Omega\left(x_{n}, k_{n}\right)\right)>\varepsilon$ for each $n$. Therefore, $\left(x^{*}, k^{*}\right)$ is an $\Omega(x, k)$-bifurcation point. The proposition is proved.

The points of $\omega(k)$ - and $\omega(x, k)$-bifurcation may be called bifurcations with the appearance of new $\omega$ limit points, and $\Omega(k)$ - and $\Omega(x, k)$-bifurcations, bifurcations with the appearance of new $\omega$-limit sets. In the first case, there exists a sequence of points $k_{n}\left(\right.$ or $\left.\left(x_{n}, k_{n}\right)\right)$ converging to a bifurcation point $k^{*}$ (or $\left.\left(x^{*}, k^{*}\right)\right)$ such that there is a point $x_{0} \in \omega\left(k^{*}\right)\left(\right.$ or $\left.\left(x_{0} \in \omega\left(x^{*}, k^{*}\right)\right)\right)$ situated from all $\omega\left(k_{n}\right)\left(\omega\left(x_{n}, k_{n}\right)\right)$ farther than at some $\varepsilon>0$. This point may be called a "new" $\omega$-limit point. In the second case, as was demonstrated, the existence of bifurcations is equivalent to the fact that there exists a sequence of points $k_{n} \in K$ (or $\left.\left(x_{n}, k_{n}\right) \in X \times K\right)$, converging to a bifurcation point $k^{*} \in K$ (or $\left(x^{*}, k^{*}\right) \in X \times K$ ), and some set $\omega\left(x_{0}, k^{*}\right) \subset \omega\left(k^{*}\right)\left(\omega\left(x_{0}, k^{*}\right) \subset \omega\left(x^{*}, k^{*}\right)\right)$ that is at the $r$-distance greater than $\gamma>0$ from all $\omega\left(k_{n}\right)\left(\omega\left(x_{n}, k_{n}\right)\right)$. This means that $\rho(x, y)>\gamma$ for all $x \in \omega\left(x_{0}, k^{*}\right)$ and $y \in \omega\left(k_{n}\right)\left(y \in \omega\left(x_{n}, k_{n}\right)\right)$. It is natural to call the set $\omega\left(x_{0}, k^{*}\right)$ the "new" $\omega$-limit set. There appears the question of whether bifurcations with the appearance of new $\omega$-limit points but without the splitting-off of new $\omega$-limit sets? A positive answer to it is given by the following example.

Example 2 (an $\omega(x, k)$ - but not an $\Omega(x, k)$-bifurcation; see Fig. 2$)$. Consider first the system defined on the cone $x^{2}+y^{2} \leq z^{2}, 0 \leq z \leq 1$ by the differential equation

$$
\dot{r}=r(2 z-r-1)^{2}-2 r(1-r)(1-z), \quad \dot{\varphi}=r \cos \varphi+1, \quad \dot{z}=-z(1-z)^{2} .
$$

As $t \rightarrow \infty$, the solutions to (7) with initial conditions $0 \leq z(0)<1,0 \leq r(0) \leq z(0)$ and arbitrary $\varphi(0)$ tend to their unique $\omega$-limit set, the equilibrium $z=r=0$. If $z(0)=1,0<r(0)<1$ then the solution tends to the circle $z=r=1$ as $t \rightarrow \infty$. If $z(0)=1, r(0)=0$ then there is only one $\omega$-limit point: $z=1, r=0$. If $z(0)=r(0)=1$ then also there is only one $\omega$-limit point: $z=1, r=1, \varphi=\pi$ (see Fig. 3). Thus,

$$
\omega\left(r_{0}, \varphi_{0}, z_{0}\right)=\left\{\begin{array}{lll}
\{(z=r=0)\} & \text { if } & z_{0}<1 \\
\{(z=r=1)\} & \text { if } & z_{0}=1, r_{0} \neq 0,1 \\
\{(z=r=1, \varphi=\pi)\} & \text { if } & z_{0}=r_{0}=1 \\
\{(z=1, r=0)\} & \text { if } & z_{0}=1, r_{0}=0
\end{array}\right.
$$

In the cone where the system is defined, consider an arbitrary sequence $\left(r_{n}, \varphi_{n}, z_{n}\right)$ tending to some point $\left(r^{*}, \varphi^{*}, 1\right)$. Suppose that $z_{n}<1$ for all $n$ and $0<r *<1$. Then the trajectory of every point $\left(r_{n}, \varphi_{n}, z_{n}\right)$ tends to the vertex of the cone, situated at the origin, while the $\omega$-limit set of the point $\left(r^{*}, \varphi^{*}, 1\right)$ is the circle $r=z=1$. These remarks clearly show that the system has $\omega(x, k)$-bifurcations. It is easy to understand that they are $\Omega(x, k)$-bifurcations. Identify now all the three equilibria as is shown in Fig. 3. Then the $\omega(x, k)$-bifurcations remain and the $\Omega(x, k)$-bifurcations disappear.

Prove the correctness of the identification. Suppose that the semiflow in a space $X$ has fixed points $x_{1}, \ldots, x_{n}$. Define a new semiflow $\tilde{f}$ as follows: As its phase space, assign the set $\widetilde{X}$ that is obtained from $X$ by removing all points $x_{1}, \ldots, x_{n}$ and adding a new point $x^{*}$. Define a metric on $\widetilde{X}$ as follows: Suppose that $x, y \in \widetilde{X}$ and $x \neq x^{*}$. Put

$$
\tilde{\rho}(x, y)=\left\{\begin{array}{lll}
\min \left\{\rho(x, y), \min _{1 \leq j \leq n} \rho\left(x, x_{j}\right)+\min _{1 \leq j \leq n} \rho\left(y, x_{j}\right)\right\} & \text { if } \quad y \neq x^{*} \\
\min _{1 \leq j \leq n} \rho\left(x, x_{j}\right) & \text { if } \quad y=x^{*} .
\end{array}\right.
$$



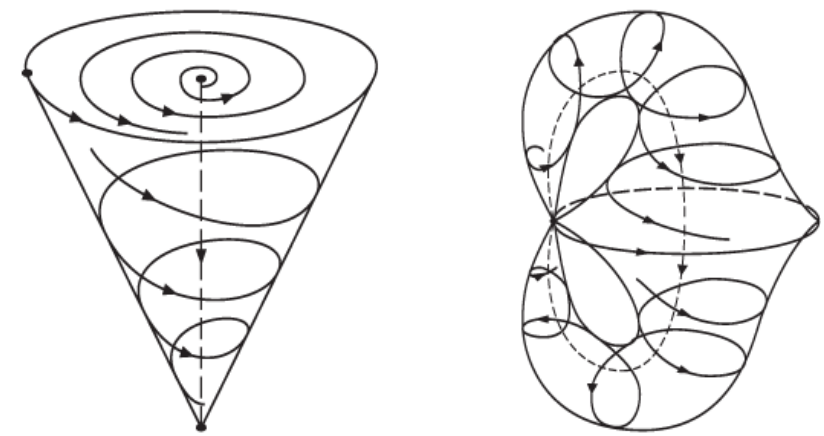

Fig. 3. $\omega(x, k)$ - but not $\Omega(x, k)$-bifurcations: the phase portrait of (7) (to the left) and the phase portrait after identification (gluing) of all fixed points (to the right)

Of course, we define $\tilde{\rho}\left(x^{*}, y\right)$ for $y \neq x^{*}$ as $\tilde{\rho}\left(y, x^{*}\right)$ and put $\tilde{\rho}\left(x^{*}, x^{*}\right)=0$. Finally, put $\tilde{f}(t, x)=f(t, x)$ if $x \in X \cap \tilde{X}$ and $\tilde{f}\left(t, x^{*}\right)=x^{*}$.

Lemma 2. The mapping $\tilde{f}$ defines a semiflow on $\tilde{X}$.

Proof. The injectivity and the semigroup property $\tilde{f}$ are obvious by the corresponding properties of $f$. If $x \in X \cap \tilde{X}, t \geq 0$, then the continuity of $\tilde{f}$ at a point $(t, x)$ follows from the fact that, in some its neighborhood, $\tilde{f}$ coincides with $f$. The continuity of $\tilde{f}$ at the point $\left(t, x^{*}\right)$ follows from the continuity of $f$ and the fact that any sequence converging to $x^{*}$ in $\widetilde{X}$ may be partitioned into a finite number of sequences each of which is either a sequence of points in $X \cap \widetilde{X}$ converging to one of the $x_{j}$ 's or a constant sequence with all elements equal to $x^{*}$ (there can be only one such sequence) plus maybe a finite set. The mapping $\tilde{f}(t, \cdot)$ is a homeomorphism since it is continuous and injective and $\widetilde{X}$ is compact. The lemma is proved.

Proposition 10. Suppose that, for every $k \in K$, every trajectory of the system lying in $\omega(k)$ is recurrent. Then the presence of $\omega(x, k)$-bifurcations is equivalent to the presence of $\Omega(x, k)$ bifurcations and the existence of $\omega(k)$-bifurcations is equivalent to the existence of $\Omega(k)$ bifurcations.

Proof. Suppose that $\left(x_{n}, k_{n}\right) \rightarrow\left(x^{*}, k^{*}\right)$, but $\omega\left(x_{n}, k_{n}\right) \nrightarrow \omega\left(x^{*}, k^{*}\right)$. Then, by Proposition 5 , there exists $\tilde{x} \in \omega\left(x^{*}, k^{*}\right)$ such that $\rho^{*}\left(\tilde{x}, \omega\left(x_{n}, k_{n}\right)\right) \neq 0$. Of course, we may assume that the inequality $\rho^{*}\left(\tilde{x}, \omega\left(x_{n}, k_{n}\right)\right)>\varepsilon$ holds for some $\varepsilon>0$ for all $n$. Let $L$ denote the set of all limit points of the sequences $\left\{y_{n}\right\}$ with $y_{n} \in \omega\left(x_{n}, k_{n}\right)$. The set $L$ is closed and $k^{*}$-invariant. Note that $\rho^{*}(\tilde{x}, L) \geq \varepsilon$. Therefore, $\omega\left(\tilde{x}, k^{*}\right) \cap L=\emptyset$, since $\omega\left(\tilde{x}, k^{*}\right)$ is a minimal set (Birkhoff's Theorem, see [12, p. 404]). This implies the existence of $\delta>0$ such that $r(\omega(\tilde{x}, k), L)>\delta$ and, hence, $r\left(\omega\left(\tilde{x}, k^{*}\right), \omega\left(x_{n}, k_{n}\right)\right)>\delta / 2$ for all sufficiently large $n$. By Proposition 7 , this implies $\Omega\left(x_{n}, k_{n}\right) \neq \Omega\left(x^{*}, k^{*}\right)$.

Thus, the presence of $\omega(x, k)$-bifurcations implies the existence of $\Omega(x, k)$-bifurcations. The converse implication is guaranteed by Proposition 8 . The proof of the second assertion coincides almost verbatim with that of the first.

Corollary 1. Suppose that $\omega(x, k)$ is minimal for every pair $(x, k) \in X \times K$. Then the two claims of Proposition 10 hold.

It suffices to observe that, by one of Birkhoff's Theorems [12, p. 402], every trajectory lying in a minimal set is recurrent.

\section{ACKNOWLEDGMENTS}

The authors are grateful to V. I. Elokhin and G. S. Yablonskii who familiarized them with the problem of slow relaxations in chemistry, and to $\mathrm{V}$. V. Ivanov for a fruitful discussion of the results. We express our heartfelt gratitude to E. P. Volokitin who created the electronic versions of the figures in this article. 


\section{REFERENCES}

1. I. M. Glazman, 'Relaxation on Surfaces with Saddle Points,” Dokl. Akad. Nauk SSSR 161 (4), 750-752 (1965).

2. M. I. Temkin, "Relaxation of the Reaction Rate in the Case of a Two-Stage Catalytic Reaction," Kinetika i Kataliz 17 (5), 1095 (1976).

3. L. P. Levchenko, N. V. Kul'kova, and M. I. Temkin, "Transient Processes under Ethylene Oxidation over Silver," Kinetika i Kataliz 17 (6), 1542 (1976).

4. A. N. Gorban', V. I. Elokhin, V. M. Cheresiz, and G. S. Yablonskii, "Possible Kinetic Causes for Slow Relaxation in Catalytic Reactions," in Unsteady State Processes in Catalysis, Vol. 1 (Inst. Catalysis, Novosibirsk, 1979), pp. 83-88.

5. A. N. Gorban', V. M. Cheresiz, V. I. Elokhin, and G. S. Yablonskii, "Slow Relaxations and Critical Phenomena in Catalytic Reactions," in Mathematical Methods in Chemistry, Vol. 2, Part 3 (Yaroslavl', 1979), pp. 20-22.

6. A. N. Gorban' and V. M. Cheresiz, "Slow Relaxations of Dynamical Systems and Bifurcations of the $\omega$-Limit Sets,” Dokl. Akad. Nauk SSSR 261 (5), 1050-1053 (1981) [Sov. Math., Dokl. 24, 645-649 (1981)].

7. A. N. Gorban' and V. M. Cheresiz, Slow Relaxations and Bifurcations of Limit Sets of Dynamical Systems, Preprint No. 17 (Comput. Center, Krasnoyarsk, 1980).

8. N. N. Semenov, Chain Reactions (Goskhimizdat, Leningrad, 1934) [in Russian].

9. S. I. Spivak, A. B. Shabat, and A. S. Shmelev, "About the Inductive Period of Chemical Reactions," in Unsteady State Processes in Catalysis, Vol. 1 (Inst. Catalysis, Novosibirsk, 1979), pp. 118-121.

10. A. D. Venttsel' and M. I. Freindlin, Fluctuations of Dynamical Systems under Small Random Perturbations (Nauka, Moscow, 1979) [in Russian].

11. J. D. Birkhoff, Dynamical Systems (Gostekhizdat, Moscow, 1941) [in Russian].

12. V. V. Nemytskii and V. V. Stepanov, Theory of Differential Equations (Gostekhizdat, Moscow, 1949) [in Russian].

13. K. S. Sibirskii, Introduction to Topological Dynamics (Akad. Nauk Moldav. SSR, Kishinev, 1970) [in Russian].

14. K. Kuratowski, Topology, Vol. 1 (PWN, Warszawa, 1961; Mir, Moscow, 1966).

15. K. Kuratowski, Topology, Vol. 2 (PWN, Warszawa, 1961; Mir, Moscow, 1969).

16. N. Bourbaki, General Topology. Main Structures (Nauka, Moscow, 1968) [in Russian].

17. Z. Nitetski, Differentiable Dynamics (The MIT Press, Cambridge, 1971; Mir, Moscow, 1975). 